\title{
Analysis of Linear Piecewise Constant Delay Systems Using a Hybrid Numerical Scheme
}

\author{
H. R. Marzban and S. M. Hoseini \\ Department of Mathematical Sciences, Isfahan University of Technology, Isfahan 8415683111, Iran \\ Correspondence should be addressed to H. R. Marzban; hmarzban@cc.iut.ac.ir
}

Received 30 April 2016; Accepted 3 July 2016

Academic Editor: Marc Gerritsma

Copyright ( $) 2016$ H. R. Marzban and S. M. Hoseini. This is an open access article distributed under the Creative Commons Attribution License, which permits unrestricted use, distribution, and reproduction in any medium, provided the original work is properly cited.

\begin{abstract}
An efficient computational technique for solving linear delay differential equations with a piecewise constant delay function is presented. The new approach is based on a hybrid of block-pulse functions and Legendre polynomials. A key feature of the proposed framework is the excellent representation of smooth and especially piecewise smooth functions. The operational matrices of delay, derivative, and product corresponding to the mentioned hybrid functions are implemented to transform the original problem into a system of algebraic equations. Illustrative examples are included to demonstrate the validity and applicability of the proposed numerical scheme.
\end{abstract}

\section{Introduction}

Delay differential equations (DDEs) naturally arise in diverse areas of science and engineering such as transmission lines, communication networks, biological models, population dynamics, and transportation systems $[1,2]$. So far, a large body of literature has been devoted to the theoretical aspects and numerical treatments of DDEs with constant delays [318]. It is known that, except for some simple cases, it is either extremely difficult or impossible to analytically solve DDEs. Accordingly, a numerical algorithm has to be adopted in most cases. The situation becomes more complicated when the time-delay is a piecewise constant function. Owing to the nature of DDEs, none of the smooth basis functions is able to properly model the inherent behavior of this class of systems. This is due to the lack of smoothness of analytical solution associated with DDEs. It should be pointed out that the approximation of a piecewise smooth function by a finite number of smooth functions often fails to converge because of the existence of the well-known Gibbs phenomenon. Consequently, a suitable basis is required to accurately model the true locations of the switching points that occur in the exact solution of a delay differential equation. It is generally assumed that the delay function is either constant or continuous. However, in some situations, time-delay is a piecewise constant function. To the best of our knowledge, few research works have been dedicated to the development of computational algorithms for solving DDEs involving piecewise constant delay $[4,19]$. Recently, Marzban and Shahsiah proposed an efficient numerical scheme for solving DDEs containing piecewise constant delay. Their method is based on a hybrid of block-pulse functions and Chebyshev polynomials. It has been demonstrated that the method implemented in [19] is effective and produces very accurate results.

In what follows, we describe some similarities and differences between our method and the procedures developed in $[15,16]$. First, the foundations of the proposed framework and those used in the previous works are based on a hybrid of block-pulse functions and Legendre polynomials. Second, the current paper is an extension of our previous ones. More specifically, the time-delay systems considered in $[15,16]$ involve constant delay, while here we investigate linear piecewise constant delay systems. Obviously, the latter systems are a general class of constant DDEs. Third, the approach employed here is based on the derivative matrix corresponding to the mentioned hybrid functions while the method implemented in our earlier works is based on the operational matrix of integration. Fourth, the operational matrix of delay associated with the piecewise constant delay systems is 
constructed. To derive this matrix, we use the same approach as that of [19]. The purpose of this paper is to introduce an efficient numerical technique for solving DDEs with a piecewise constant delay. It should be emphasized that the analytical solutions of DDEs cannot be obtained solely either by blockpulse functions or by Legendre polynomials. Combining block-pulse functions and Legendre polynomials allows one to simultaneously make use of the best properties of the two mentioned bases. It is worth noting that the value of $N$, the order of block-pulse functions, plays an essential role in modelling of the problem under consideration. Indeed, by selecting the suitable value of $N$, we are able to correctly determine the exact locations of the switching points that occur in the solution associated with a piecewise constant delay system. The excellent properties of hybrid functions together with the operational matrices of delay, derivative, and product are then utilized to transform the delay differential equation under investigation into a system of algebraic equations whose solution is much easier than the original one.

The rest of the paper is organized as follows. In Section 2, the basic properties of hybrid of block-pulse functions and Legendre polynomials are presented. In Section 3, the operational matrices of derivative, product, and delay are presented. Section 4 is devoted to the problem statement and its approximation. In Section 5, three examples are tested to show the efficiency and accuracy of the proposed numerical scheme.

\section{Hybrid Functions}

Hybrid functions $\phi_{n m}(t), n=1,2, \ldots, N, m=0,1, \ldots, M$, are defined on the interval $\left[0, t_{f}\right)$ as [15]

$$
\begin{aligned}
& \phi_{n m}(t) \\
& = \begin{cases}P_{m}\left(\frac{2 N}{t_{f}} t-2 n+1\right), & t \in\left[\left(\frac{n-1}{N}\right) t_{f}, \frac{n}{N} t_{f}\right), \\
0, & \text { otherwise, }\end{cases}
\end{aligned}
$$

where $n$ and $m$ are the orders of block-pulse functions and Legendre polynomials, respectively. Here, $P_{m}(t)$ are the wellknown Legendre polynomials of order $m$ which are orthogonal on the interval $[-1,1]$ and satisfy the following recursive formula [20]:

$$
\begin{aligned}
P_{0}(t) & =1, \\
P_{1}(t) & =t \\
P_{m+1}(t) & =\left(\frac{2 m+1}{m+1}\right) t P_{m}(t)-\left(\frac{m}{m+1}\right) P_{m-1}(t), \\
& m=1,2,3, \ldots .
\end{aligned}
$$

Since $\phi_{n m}(t)$ consists of block-pulse functions and Legendre polynomials, which are both complete and orthogonal, the set of the hybrid of block-pulse functions and Legendre polynomials is a complete orthogonal set in the Hilbert space $\mathscr{L}^{2}\left[0, t_{f}\right)$.

\section{Operational Matrices}

In this section, we first present the operational matrix of derivative based on the weak representation of the derivative operator. We then state the operational matrices of delay and product corresponding to the proposed hybrid functions.

A function $u \in \mathscr{L}^{2}\left[0, t_{f}\right)$ can be approximated by the hybrid functions as follows:

$$
u(t) \simeq \sum_{n=1}^{N} \sum_{m=0}^{M} \widehat{u}_{n m} \phi_{n m}(t)=\Phi^{T}(t) U,
$$

where

$$
U=\left[\widehat{u}_{10}, \ldots, \widehat{u}_{1 M}, \widehat{u}_{20}, \ldots, \widehat{u}_{2 M}, \ldots, \widehat{u}_{N 0}, \ldots, \widehat{u}_{N M}\right]^{T}
$$

is the vector of coefficients and

$$
\begin{gathered}
\Phi(t)=\left[\phi_{10}(t), \ldots, \phi_{1 M}(t), \phi_{20}(t), \ldots, \phi_{2 M}(t), \ldots,\right. \\
\left.\phi_{N 0}(t), \ldots, \phi_{N M}(t)\right]^{T} .
\end{gathered}
$$

The coefficients $\widehat{u}_{n m}, n=1,2, \ldots, N, m=0,1, \ldots, M$, are obtained by the following formula:

$$
\widehat{u}_{n m}=\frac{N(2 m+1)}{t_{f}} \int_{((n-1) / N) t_{f}}^{(n / N) t_{f}} u(t) \phi_{n m}(t) d t .
$$

3.1. The Operational Matrix of Derivative. We approximate the derivative of $u$ by the derivative of (3); that is,

$$
\frac{d}{d t} u(t) \simeq \frac{d}{d t}\left(\sum_{n=1}^{N} \sum_{m=0}^{M} \widehat{u}_{n m} \phi_{n m}(t)\right) .
$$

The right hand side of the preceding equation can be represented in terms of hybrid functions as

$$
\begin{aligned}
& \frac{d}{d t}\left(\sum_{n=1}^{N} \sum_{m=0}^{M} \widehat{u}_{n m} \phi_{n m}(t)\right)=\sum_{n=1}^{N} \sum_{m=0}^{M} \widehat{s}_{n m} \phi_{n m}(t) \\
& \quad=\Phi^{T}(t) S,
\end{aligned}
$$

where

$$
S=\left[\widehat{s}_{10}, \ldots, \widehat{s}_{1 M}, \widehat{s}_{20}, \ldots, \widehat{s}_{2 M}, \ldots, \widehat{s}_{N 0}, \ldots, \widehat{s}_{N M}\right]^{T} .
$$

The relationship between the two vectors $U$ and $S$ is expressed by

$$
S=\mathrm{D} U
$$

where $N(M+1) \times N(M+1)$ matrix $\mathbf{D}$ is the operational matrix of derivative.

The structure of the operational matrix of derivative $\mathbf{D}$ is given by [21].

In the case $N=1$,

$$
\mathbf{D}=A,
$$


in the case $N=2$,

$$
\mathbf{D}=\left(\begin{array}{ll}
A_{1} & B_{1} \\
C_{N} & A_{N}
\end{array}\right),
$$

and, in the case $N \geq 3$,

$$
\mathbf{D}=\left(\begin{array}{ccccc}
A_{1} & B_{1} & & & \\
C_{2} & A_{2} & B_{2} & & \\
& \ddots & \ddots & \ddots & \\
& & C_{N-1} & A_{N-1} & B_{N-1} \\
& & & C_{N} & A_{N}
\end{array}\right),
$$

where, for $m, j=0,1, \ldots, M$, we set

$$
\begin{aligned}
& {[A]_{m j} }=\frac{N(2 m+1)}{t_{f}}\left(1-(-1)^{m+j}-q_{m j}\right), \\
& {\left[A_{1}\right]_{m j}=} \frac{N(2 m+1)}{t_{f}}\left(\frac{1}{2}-(-1)^{m+j}-q_{m j}\right), \\
& {\left[A_{n}\right]_{m j}=\frac{N(2 m+1)}{t_{f}}\left(\frac{1}{2}-\frac{(-1)^{m+j}}{2}-q_{m j}\right), } n=2,3, \ldots, N-1, \\
& {\left[A_{N}\right]_{m j}=\frac{N(2 m+1)}{t_{f}}\left(1-\frac{(-1)^{m+j}}{2}-q_{m j}\right), } \\
& {\left[B_{n}\right]_{m j}=\frac{N(2 m+1)}{t_{f}}\left(\frac{(-1)^{j}}{2}\right), } \\
& q_{m j}=\left\{\begin{array}{l}
2, \quad \text { if } \exists k \in \mathbb{Z} ; m-j=2 k+1>0, \\
0, \quad \text { otherwise. }
\end{array}\right] \\
& {\left[C_{m j}=\frac{N(2 m+1)}{t_{f}}\left(-\frac{(-1)^{m}}{2}\right), \quad n=2,3, \ldots, N,\right.}
\end{aligned}
$$

3.2. The Operational Matrix of Product. Let $W$ be an arbitrary vector of order $N(M+1) \times 1$. Then, the expression $\Phi(t) \Phi^{T}(t) W$ can be expanded in terms of hybrid functions as follows:

$$
\Phi(t) \Phi^{T}(t) W \simeq \widetilde{W} \Phi(t)
$$

in which $\widetilde{W}$ is a matrix of order $N(M+1) \times N(M+1)$. This matrix is called the operational matrix of product and has the following structure:

$$
\widetilde{W}=\left[\begin{array}{ccccc}
\widetilde{W}_{1} & O & O & \cdots & O \\
O & \widetilde{W}_{2} & O & \cdots & O \\
O & O & \widetilde{W}_{3} & \cdots & O \\
\vdots & \vdots & \vdots & \ddots & \vdots \\
O & O & O & \cdots & \widetilde{W}_{N}
\end{array}\right],
$$

where $\widetilde{W}_{i}, i=1,2,3, \ldots, N$, are $(M+1) \times(M+1)$ matrices given in [15].

3.3. The Operational Matrix of Delay. The goal of this subsection is to determine the operational matrix of delay associated with the developed hybrid functions. For this purpose, let

$$
\Phi(t-\tau(t))=\mathscr{D} \Phi(t)
$$

in which $\tau(t)$ is defined by

$$
\tau(t)= \begin{cases}\tau_{1}, & T_{0} \leq t<T_{1} \\ \tau_{2}, & T_{1} \leq t<T_{2} \\ \vdots & \vdots \\ \tau_{r}, & T_{r-1} \leq t \leq T_{r}\end{cases}
$$

where $0=T_{0}<T_{1}<T_{2}<\cdots<T_{r-1}<T_{r}=t_{f}$ and $\tau_{i}$, $i=1, \ldots, r$, are known constants. Furthermore, assume that $I=\left\{i: \tau_{i} \neq 0\right\}$ is a nonempty set, $\tau_{i}, i=1,2, \ldots, r$, are nonnegative rational numbers, and $T_{i} \in \mathbb{Q}$ for $i=0,1, \ldots, r$.

To obtain the operational matrix of delay corresponding to the proposed hybrid functions, we apply an approach analogous to the one devised in [19]. To do this, we first divide the time interval $\left[0, t_{f}\right]$ into $N$ subintervals of the same length where the value of $N$ is obtained in the following manner.

Define $\gamma$ as the smallest positive integer number in such a way that

$$
\begin{aligned}
\gamma \tau_{i} & \in \mathbb{Z}, \quad i \in I, \\
\gamma T_{j} \in \mathbb{Z}, & j=0,1, \ldots, r .
\end{aligned}
$$

Suppose that $|I|=l$. Next, we choose $\lambda$ as the greatest common divisor of the integers $\gamma \tau_{i}$ and $\gamma T_{j}, i \in I, j=0,1$, $\ldots, r$; that is,

$$
\lambda=\operatorname{g.c.d}\left(\gamma \tau_{1}, \gamma \tau_{2}, \ldots, \gamma \tau_{l}, \gamma T_{1}, \ldots, \gamma T_{r}\right) .
$$

Let

$$
N= \begin{cases}\frac{\gamma}{\lambda} t_{f}, & \text { if } \frac{\gamma}{\lambda} t_{f} \in \mathbb{Z}, \\ {\left[\frac{\gamma}{\lambda} t_{f}\right]+1,} & \text { otherwise }\end{cases}
$$

where $\left[(\gamma / \lambda) t_{f}\right]$ denotes the greatest integer value less than or equal to $(\gamma / \lambda) t_{f}$. It should be noted that $N$ is chosen in such a way that the number of subintervals is minimized. As a consequence, we obtain the following subintervals:

$$
[0, h],[h, 2 h], \ldots,[(N-1) h, N h],
$$

where $h=\lambda / \gamma=t_{f} / N$. Now, from (20) and (22) we can find the integer numbers $k_{i}$ and $l_{j}$, such that

$$
\begin{aligned}
\tau_{i} & =k_{i} h, \quad i \in I, \\
T_{j} & =l_{j} h, \quad j=0,1, \ldots, r .
\end{aligned}
$$


Note that $k_{i}=0$, for $i \notin I, 1 \leq i \leq r$. Therefore, we can rewrite $\tau(t)$ as follows:

$$
\tau(t)= \begin{cases}k_{1} h, & l_{0} h \leq t<l_{1} h, \\ k_{2} h, & l_{1} h \leq t<l_{2} h, \\ \vdots & \vdots \\ k_{r} h, & l_{r-1} h \leq t \leq l_{r} h .\end{cases}
$$

For convenience, we define $k(i)$ as

$$
k(i)= \begin{cases}k_{1}, & 0 \leq i \leq l_{1} \\ k_{2}, & l_{1}<i \leq l_{2} \\ \vdots & \vdots \\ k_{r}, & l_{r-1}<i \leq l_{r} .\end{cases}
$$

Therefore, the problem is reduced to find the operational matrix of delay for the following delay function:

$$
\Phi(t-\tau(t))= \begin{cases}\Phi(t-k(1) h), & 0 \leq t<t_{1}, \\ \Phi(t-k(2) h), & t_{1} \leq t<t_{2} \\ \vdots & \vdots \\ \Phi(t-k(N) h), & t_{N-1} \leq t<t_{N}\end{cases}
$$

where

$$
t_{i}=l_{i} h, \quad i=1,2, \ldots, N .
$$

In order to find the matrix $\mathscr{D}$, we first derive the matrix $D_{i}$ for $i=1,2, \ldots, N$, so that the following relation holds:

$$
\Phi(t-k(i) h)=D_{i} \Phi(t), \quad t_{i-1} \leq t<t_{i} .
$$

With the use of (1), it is worth noting that, in the case of $t_{i-1} \leq t<t_{i}$, the only functions with nonzero values are $\phi_{(i-k(i)) m}(t-k(i) h)$, for $m=0,1, \ldots, M$. Since

$$
\phi_{(i-k(i)) m}(t-k(i) h)=\phi_{i m}(t), \quad m=0,1, \ldots, M,
$$

by expanding $\phi_{(i-k(i)) m}(t-k(i) h)$ in terms of $\phi_{i m}(t)$, we get the $(M+1) \times(M+1)$ identity matrix as the corresponding coefficients. Therefore, we have

$$
D_{i}=S_{i} \otimes I_{M+1}, \quad i=1,2, \ldots, N,
$$

where $I_{M+1}$ is the $(M+1)$-dimensional identity matrix and $S_{i}$ is an $N \times N$ matrix in which the only nonzero entry is equal to one and located at the $(i-k(i))$ th row and $i$ th column.

Remark 1. If $i-k(i) \leq 0$, then $S_{i}$ is a zero matrix of order $N \times N$.

As a consequence, if we expand $\Phi(t-\tau(t))$ in terms of hybrid functions $\Phi(t)$, we deduce that

$$
\mathscr{D}=D_{1}+D_{2}+\cdots+D_{N}
$$

To illustrate the derivation process of the operational matrix of delay associated with the mentioned hybrid functions, we present an example. Define

$$
\tau(t)= \begin{cases}\frac{1}{6}, & 0 \leq t<\frac{1}{3}, \\ \frac{1}{2}, & \frac{1}{3} \leq t \leq 1 .\end{cases}
$$

It is easily verified that $N=6$. As a result, we get

$$
k(i)= \begin{cases}1, & 1 \leq i \leq 2 \\ 3, & 3 \leq i \leq 6\end{cases}
$$

For $i=1$ and $i=3$, we conclude that $i-k(i)=0$. Therefore, $S_{1}$ and $S_{3}$ are zero matrices of order $6 \times 6$. If $i=2$, it follows that $i-k(i)=1$. Consequently, $S_{2}$ is a $6 \times 6$ matrix in which the only nonzero entry is equal to one and located at the 1st row and 2 nd column. If $i=4$, then we deduce that $i-k(i)=1$. Hence, $S_{4}$ is a $6 \times 6$ matrix in which the only nonzero entry is equal to one and located at the 1 st row and 4 th column. A similar argument can be used for $i=5$ and $i=6$ to specify matrices $S_{5}$, and $S_{6}$. Using the above comments, we obtain

$$
D_{1}=S_{1} \otimes I_{M}=\left[\begin{array}{llllll}
0 & 0 & 0 & 0 & 0 & 0 \\
0 & 0 & 0 & 0 & 0 & 0 \\
0 & 0 & 0 & 0 & 0 & 0 \\
0 & 0 & 0 & 0 & 0 & 0 \\
0 & 0 & 0 & 0 & 0 & 0 \\
0 & 0 & 0 & 0 & 0 & 0
\end{array}\right],
$$$$
D_{2}=S_{2} \otimes I_{M}=\left[\begin{array}{cccccc}
0 & I_{M} & 0 & 0 & 0 & 0 \\
0 & 0 & 0 & 0 & 0 & 0 \\
0 & 0 & 0 & 0 & 0 & 0 \\
0 & 0 & 0 & 0 & 0 & 0 \\
0 & 0 & 0 & 0 & 0 & 0 \\
0 & 0 & 0 & 0 & 0 & 0
\end{array}\right] \text {, }
$$$$
D_{3}=S_{3} \otimes I_{M}=\left[\begin{array}{llllll}
0 & 0 & 0 & 0 & 0 & 0 \\
0 & 0 & 0 & 0 & 0 & 0 \\
0 & 0 & 0 & 0 & 0 & 0 \\
0 & 0 & 0 & 0 & 0 & 0 \\
0 & 0 & 0 & 0 & 0 & 0 \\
0 & 0 & 0 & 0 & 0 & 0
\end{array}\right] \text {, }
$$

$$
D_{4}=S_{4} \otimes I_{M}=\left[\begin{array}{cccccc}
0 & 0 & 0 & I_{M} & 0 & 0 \\
0 & 0 & 0 & 0 & 0 & 0 \\
0 & 0 & 0 & 0 & 0 & 0 \\
0 & 0 & 0 & 0 & 0 & 0 \\
0 & 0 & 0 & 0 & 0 & 0 \\
0 & 0 & 0 & 0 & 0 & 0
\end{array}\right] \text {, }
$$




$$
\begin{gathered}
D_{5}=S_{5} \otimes I_{M}=\left[\begin{array}{llllcc}
0 & 0 & 0 & 0 & 0 & 0 \\
0 & 0 & 0 & 0 & I_{M} & 0 \\
0 & 0 & 0 & 0 & 0 & 0 \\
0 & 0 & 0 & 0 & 0 & 0 \\
0 & 0 & 0 & 0 & 0 & 0 \\
0 & 0 & 0 & 0 & 0 & 0
\end{array}\right], \\
D_{6}=S_{6} \otimes I_{M}=\left[\begin{array}{lllllc}
0 & 0 & 0 & 0 & 0 & 0 \\
0 & 0 & 0 & 0 & 0 & 0 \\
0 & 0 & 0 & 0 & 0 & I_{M} \\
0 & 0 & 0 & 0 & 0 & 0 \\
0 & 0 & 0 & 0 & 0 & 0 \\
0 & 0 & 0 & 0 & 0 & 0
\end{array}\right] .
\end{gathered}
$$

Consequently

$$
\mathscr{D}=\sum_{i=1}^{6} D_{i}=\left[\begin{array}{cccccc}
0 & I_{M} & 0 & I_{M} & 0 & 0 \\
0 & 0 & 0 & 0 & I_{M} & 0 \\
0 & 0 & 0 & 0 & 0 & I_{M} \\
0 & 0 & 0 & 0 & 0 & 0 \\
0 & 0 & 0 & 0 & 0 & 0 \\
0 & 0 & 0 & 0 & 0 & 0
\end{array}\right] .
$$

\section{Problem Statement and Its Approximation}

4.1. Piecewise Constant Delay Systems. Consider the linear time-varying piecewise constant delay system described by

$$
\begin{aligned}
& \dot{x}(t)=A(t) x(t)+B(t) x(t-\tau(t))+C(t) u(t), \\
& x(t)=\varphi(t), \quad t \leq 0, \\
& \tau(t)= \begin{cases}\tau_{1}, & T_{0} \leq t<T_{1}, \\
\tau_{2}, & T_{1} \leq t<T_{2}, \\
\vdots & \vdots \\
\tau_{r}, & T_{r-1} \leq t \leq T_{r},\end{cases}
\end{aligned}
$$

where $x(t) \in \mathbb{R}^{p}, u(t) \in \mathbb{R}^{q}$, and $A(t), B(t)$, and $C(t)$ are matrices with appropriate dimensions. The problem is to find $x(t), t \in\left[0, t_{f}\right]$, satisfying (37)-(39).

4.2. Approximation Using Hybrid Functions. We approximate the system dynamics by hybrid functions as follows. Let

$$
\begin{aligned}
& x(t)=\left[x_{1}(t), x_{2}(t), \ldots, x_{p}(t)\right]^{T}, \\
& u(t)=\left[u_{1}(t), u_{2}(t), \ldots, u_{q}(t)\right]^{T} .
\end{aligned}
$$

Using (3), each $x_{i}(t)$ and each $u_{j}(t), i=1,2, \ldots, p, j=$ $1,2, \ldots, q$, can be written in terms of hybrid functions as follows:

$$
\begin{aligned}
& P_{M}^{N} x_{i}=\sum_{n=1}^{N} \sum_{m=0}^{M} \widehat{x}_{i n m} \phi_{n m}(t)=\Phi^{T}(t) X_{i}, \\
& P_{M}^{N} u_{j}=\sum_{n=1}^{N} \sum_{m=0}^{M} \widehat{u}_{j n m} \phi_{n m}(t)=\Phi^{T}(t) U_{j} .
\end{aligned}
$$

From (40), it follows that

$$
\begin{aligned}
& P_{M}^{N} x(t)=\left(I_{p} \otimes \Phi^{T}(t)\right) X, \\
& P_{M}^{N} u(t)=\left(I_{q} \otimes \Phi^{T}(t)\right) U,
\end{aligned}
$$

where $I_{p}$ and $I_{q}$ are the $p \times p$ and $q \times q$ identity matrices, respectively, and $\otimes$ denotes the Kronecker product [22]. It should be noted that $X$ and $U$ are vectors of orders $p N(M+$ $1) \times 1$ and $q N(M+1) \times 1$, respectively, given by

$$
\begin{aligned}
& X=\left[X_{1}^{T}, X_{2}^{T}, \ldots, X_{p}^{T}\right]^{T}, \\
& U=\left[U_{1}^{T}, U_{2}^{T}, \ldots, U_{q}^{T}\right]^{T} .
\end{aligned}
$$

Also

$$
\varphi(t-\tau(t))=\left(I_{p} \otimes \Phi^{T}(t)\right) R,
$$

where $R$ is a vector of order $p N(M+1) \times 1$ defined by

$$
R=\left[R_{1}^{T}, R_{2}^{T}, \ldots, R_{p}^{T}\right]^{T} .
$$

Similarly

$$
\begin{aligned}
& A(t)=A^{T}\left(I_{p} \otimes \Phi(t)\right), \\
& B(t)=B^{T}\left(I_{p} \otimes \Phi(t)\right), \\
& C(t)=C^{T}\left(I_{q} \otimes \Phi(t)\right),
\end{aligned}
$$

where $A, B$, and $C$ are matrices of orders $p N(M+1) \times p$, $p N(M+1) \times p$, and $q N(M+1) \times p$, respectively. The delay vector $x(t-\tau(t))$ can also be expanded in terms of hybrid functions as

$$
\begin{aligned}
P_{M}^{N} x(t-\tau(t))= & \left(I_{p} \otimes \Phi^{T}(t)\right) R \\
& +\left(I_{p} \otimes \Phi^{T}(t)\right)\left(I_{p} \otimes \mathscr{D}^{T}\right) X, \\
& 0 \leq t \leq t_{f},
\end{aligned}
$$

where $\mathscr{D}$ is the operational matrix of delay described by (18).

Now, using (16), we have

$$
\begin{aligned}
A(t) x(t) & =A^{T}\left(I_{p} \otimes \Phi(t)\right)\left(I_{p} \otimes \Phi^{T}(t)\right) X \\
& =\left(I_{p} \otimes \Phi^{T}(t)\right) \widetilde{A}^{T} X, \\
C(t) u(t) & =C^{T}\left(I_{q} \otimes \Phi(t)\right)\left(I_{q} \otimes \Phi^{T}(t)\right) U \\
& =\left(I_{p} \otimes \Phi^{T}(t)\right) \widetilde{C}^{T} U,
\end{aligned}
$$


where $\widetilde{A}$ and $\widetilde{C}$ can be calculated in a way similar to the construction method of matrix $\widetilde{U}$ given by (17). Moreover,

$$
\begin{aligned}
& B(t) x(t-\tau(t)) \\
& =B^{T}\left(I_{p} \otimes \Phi(t)\right)\left(I_{p} \otimes \Phi^{T}(t)\right)\left[R+\left(I_{p} \otimes \mathscr{D}^{T}\right) X\right] .
\end{aligned}
$$

Consequently

$$
\begin{aligned}
B(t) & x(t-\tau(t)) \\
= & \left(I_{p} \otimes \Phi^{T}(t)\right) \widetilde{B}^{T}\left[R+\left(I_{p} \otimes \mathscr{D}^{T}\right) X\right] .
\end{aligned}
$$

Now, using the operational matrix of derivative and substituting (44)-(50) in (37), we get

$$
\begin{aligned}
\left(I_{p} \otimes\right. & \left.\Phi^{T}(t)\right)\left(I_{p} \otimes \mathbf{D}\right) X \\
= & \left(I_{p} \otimes \Phi^{T}(t)\right) \widetilde{A}^{T} X+\left(I_{p} \otimes \Phi^{T}(t)\right) \widetilde{C}^{T} U \\
& +\left(I_{p} \otimes \Phi^{T}(t)\right) \widetilde{B}^{T}\left[R+\left(I_{p} \otimes \mathscr{D}^{T}\right) X\right] .
\end{aligned}
$$

Because the elements of $\Phi(t)$ are linearly independent functions over the interval $\left[0, t_{f}\right]$, it follows that

$$
\left(I_{p} \otimes \mathbf{D}\right) X=\widetilde{A}^{T} X+\widetilde{C}^{T} U+\widetilde{B}^{T}\left[R+\left(I_{p} \otimes \mathscr{D}^{T}\right) X\right] .
$$

$$
x(t)= \begin{cases}1+t+t^{2}, & 0 \leq t<0.2, \\ \frac{62341}{62500}+t+t^{2}+\frac{7}{25} t^{3}+\frac{3}{20} t^{4}+\frac{1}{5} t^{5}, & 0.2 \leq t<0.3, \\ \frac{1006717}{1000000}+t+t^{2}, & 0.3 \leq t<0.7, \\ \frac{2720369}{3000000}+t+t^{2}+\frac{79}{300} t^{3}-\frac{1}{10} t^{4}+\frac{1}{5} t^{5}, & 0.7 \leq t<0.9, \\ \frac{76256550101}{840000000000}+t+t^{2}+\frac{693817}{3000000} t^{3}+\frac{459}{40000} t^{4}+\frac{167}{5000} t^{5}+\frac{7}{50} t^{6}-\frac{11}{140} t^{7}+\frac{1}{40} t^{8}, & 0.9 \leq t \leq 1 .\end{cases}
$$

To solve this problem by the method developed in the present paper, we first determine the value of $N$, the required number of subintervals, with the use of (22). For this problem, we choose $N=10$. Also, we select $M=8$. Let

$$
x(t)=X^{T} \Phi(t) .
$$

By expanding $u(t)$ and $t^{2}$ in terms of hybrid functions, we get

$$
\begin{gathered}
u(t)=\left[\frac{11}{10}, \frac{1}{10}, 0,0,0,0,0,0,0, \frac{13}{10}, \frac{1}{10}, 0,0,0,0,0,0,0,\right. \\
\frac{3}{2}, \frac{1}{10}, 0,0,0,0,0,0,0, \frac{17}{10}, \frac{1}{10}, 0,0,0,0,0,0,0, \frac{19}{10}, \frac{1}{10}, \\
0,0,0,0,0,0,0, \frac{21}{10}, \frac{1}{10}, 0,0,0,0,0,0,0, \frac{23}{10}, \frac{1}{10}, 0,0,0,0, \\
\quad 0,0,0, \frac{5}{2}, \frac{1}{10}, 0,0,0,0,0,0,0, \frac{27}{10}, \frac{1}{10}, 0,0,0,0,0,0,0,0,
\end{gathered}
$$

The exact solution to this problem is given by [19]

$$
\left.\frac{29}{10}, \frac{1}{10}, 0,0,0,0,0,0,0\right] \Phi(t)=U^{T} \Phi(t)
$$

Therefore, the original problem is transformed into a tem of linear algebraic equations. The aforementioned sysby the well-known Tau method $[20,23]$ for the unknown vector $X$.

Example 1. Consider the time-varying piecewise constant delay system

$$
\tau(t)= \begin{cases}0.2, & 0 \leq t<0.3 \\ 0.7, & 0.3 \leq t<1\end{cases}
$$

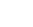

$$
\text { In this section, three examples are investigated to evaluate the }
$$
performance of the method.

$t^{2}=\left[\frac{1}{300}, \frac{1}{200}, \frac{1}{600}, 0,0,0,0,0,0, \frac{7}{300}, \frac{3}{200}, \frac{1}{600}, 0,0\right.$,

$0,0,0,0, \frac{19}{300}, \frac{1}{40}, \frac{1}{600}, 0,0,0,0,0,0, \frac{37}{300}, \frac{7}{200}, \frac{1}{600}$,

$0,0,0,0,0,0, \frac{61}{300}, \frac{9}{200}, \frac{1}{600}, 0,0,0,0,0,0, \frac{91}{300}, \frac{11}{200}$,

$\frac{1}{600}, 0,0,0,0,0,0, \frac{127}{300}, \frac{13}{200}, \frac{1}{600}, 0,0,0,0,0,0, \frac{169}{300}$, 
Substituting (58)-(60) in (53) yields

$$
\mathbf{D} X=\tilde{e}^{T} \mathscr{D}^{T} X+U,
$$

with the following initial condition:

$$
1=\sum_{m=0}^{M}(-1)^{m} X_{m},
$$

where $\mathscr{D}$ is the delay operational matrix described by (18). By solving the resulting algebraic system, we can find the vector $X$ as

$$
\begin{aligned}
X= & {\left[\frac{79}{75}, \frac{11}{200}, \frac{1}{600}, 0,0,0,0,0,0,0, \frac{88}{75}, \frac{13}{200}, \frac{1}{600}, 0,0,\right.} \\
& 0,0,0,0,0, \frac{658097}{500000}, \frac{109663}{1400000}, \frac{6059}{2800000}, \frac{1}{36000}, \\
& \frac{1}{1750000}, \frac{1}{126000000}, 0,0,0,0, \frac{4440151}{3000000}, \frac{17}{200}, \frac{1}{600}, \\
& 0,0,0,0,0,0,0, \frac{4980151}{3000000}, \frac{19}{200}, \frac{1}{600}, 0,0,0,0,0,0,0, \\
& \frac{5580151}{3000000}, \frac{21}{200}, \frac{1}{600}, 0,0,0,0,0,0,0, \frac{6240151}{3000000}, \frac{23}{200}, \\
& \frac{1}{600}, 0,0,0,0,0,0,0, \frac{4695907}{2000000}, \frac{433113}{2800000}, \frac{14701}{4200000}, \\
& \frac{49}{900000}, \frac{13}{14000000}, \frac{1}{126000000}, 0,0,0,0, \frac{3215527}{1200000}, \\
& \frac{2484429}{14000000}, \frac{691}{168000}, \frac{77}{1125000}, \frac{3}{2800000},
\end{aligned}
$$

$$
\begin{aligned}
& \frac{1}{126000000}, 0,0,0,0, \frac{1542687364553}{504000000000}, \\
& \frac{57254691267}{280000000000}, \frac{27335101823}{5544000000000}, \frac{360730541}{3960000000000}, \\
& \frac{12971011}{8008000000000}, \frac{10861}{504000000000}, \\
& \frac{7481}{27720000000000}, \frac{1}{308000000000}, \\
& \left.\frac{1}{51480000000000}, 0\right]^{T} .
\end{aligned}
$$

With the use of (58), we would obtain the same value as the exact value of $x(t)$.

Example 2. Consider the following piecewise constant delay system [13]:

$$
\begin{aligned}
& \dot{x}(t)=-5 x(t)-5 x(t-\tau(t))+2 u(t), \quad 0 \leq t \leq 2, \\
& x(0)=1 \\
& u(t)=1, \quad t \geq 0 \\
& \tau(t)= \begin{cases}0, & 0 \leq t<0.8 \\
0.3, & 0.8 \leq t<1.4 \\
0.6, & 1.4 \leq t<1.7 \\
0.9, & 1.7 \leq t \leq 2\end{cases}
\end{aligned}
$$

The analytical solution to this problem is presented by [13]

$$
x(t)= \begin{cases}0.2+0.8 e^{-10 t}, & 0 \leq t<0.8 \\ 0.2+0.8 e^{3-10 t}+0.8 e^{-4}\left(1-e^{3}\right) e^{-5 t}, & 0.8 \leq t<1.1 \\ 0.2+0.8 e^{5-10 t}-4 e^{-2.5}\left(1-e^{3}\right) t e^{-5 t}+0.8\left(1-e^{3}\right)\left(6.5 e^{-2.5}+e^{-4}\right) e^{-5 t}, & 1.1 \leq t<1.4 \\ 0.2+0.8 e^{9-10 t}-4 e^{-1}\left(1-e^{3}\right) t e^{-5 t}+0.8\left(1-e^{3}\right)\left(8 e^{-1}-0.5 e^{-2.5}+e^{-4}\right) e^{-5 t}, & 1.4 \leq t \leq 1.7 \\ 0.2+0.8 e^{12-10 t}-4 e^{0.5}\left(1-e^{3}\right) t e^{-5 t}+0.8\left(1-e^{3}\right)\left(9.5 e^{0.5}-0.5 e^{-1}-0.5 e^{-2.5}+e^{-4}\right) e^{-5 t}, & 1.7 \leq t \leq 2 .\end{cases}
$$

To solve this problem by the proposed approach, we first determine the value of $N$. With the use of (22), we take $N=$ 20 . In addition, let $M$ be an arbitrary positive integer number. Define

$$
E=\max \left\{\left|x_{e}(t)-x(t)\right|: 0 \leq t \leq 2\right\},
$$

where $x(t)$ and $x_{e}(t)$ denote the approximate solution obtained by the present method and the exact solution, respectively. In Table 1, the maximum absolute error of $x(t)$ corresponding to $N=20$ and different values of $M$ are summarized. This table shows that there is an excellent agreement between the analytical and approximate solutions. After choosing $N$, small values for $M$ are needed to achieve a satisfactory approximation.

Example 3. As a more complicated problem, consider the following nonlinear piecewise constant delay system:

$$
\begin{aligned}
& \dot{x}(t)=t x(t-\tau(t))+t x^{2}(t-\tau(t))+x^{3}(t-\tau(t)) \\
& +u(t), \quad 0 \leq t \leq 1, \\
& x(0)=1 \text {, } \\
& x(t)=-1, \quad t<0,
\end{aligned}
$$


TABLE 1: Results of maximum error for Example 2.

\begin{tabular}{lc}
\hline Present method & $E$ \\
\hline$M=2$ & $2.05 e-02$ \\
$M=4$ & $2.64 e-05$ \\
$M=6$ & $2.87 e-08$ \\
$M=8$ & $2.12 e-11$ \\
$M=10$ & $1.09 e-14$ \\
$M=12$ & $4.11 e-18$ \\
$M=14$ & $1.17 e-21$ \\
\hline
\end{tabular}

$$
\begin{gathered}
u(t)= \begin{cases}1, & 0 \leq t<0.8, \\
-1, & 0.8 \leq t \leq 1,\end{cases} \\
\tau(t)= \begin{cases}0.4, & 0 \leq t<0.8, \\
0.8, & 0.8 \leq t<1 .\end{cases}
\end{gathered}
$$

The exact solution to this problem is described by

$$
x(t)= \begin{cases}1, & 0 \leq t<0.4, \\ \frac{1}{25}+2 t+t^{2}, & 0.4 \leq t<0.8 \\ \frac{41}{25}+t^{2}, & 0.8 \leq t \leq 1 .\end{cases}
$$

Although the above problem is a nonlinear delay differential equation, the method developed in the current paper is applicable. To employ the procedure described in Section 4, we first select $N=5$. Moreover, we choose $M=2$. Let

$$
x(t)=X^{T} \Phi(t) .
$$

By expressing $u(t), t$, and $\varphi(t-\tau(t))$ in terms of hybrid functions, we obtain

$$
\begin{aligned}
u(t) & =[1,0,0,1,0,0,1,0,0,1,0,0,-1,0,0] \Phi(t) \\
= & U^{T} \Phi(t) \\
t= & {\left[\frac{1}{10}, \frac{1}{10}, 0, \frac{3}{10}, \frac{1}{10}, 0, \frac{1}{2}, \frac{1}{10}, 0, \frac{7}{10}, \frac{1}{10}, 0, \frac{9}{10}, \frac{1}{10}, 0\right] } \\
& \cdot \Phi(t)=A^{T} \Phi(t) \\
\varphi(t & -\tau(t))=[-1,0,0,-1,0,0,0,0,0,0,0,0,0,0,0] \\
& \cdot \Phi(t)=R^{T} \Phi(t) .
\end{aligned}
$$

Substituting (73)-(75) in (67) implies

$$
\mathbf{D} X=\widetilde{A}^{T} K+\widetilde{A}^{T} \widetilde{K}^{T} K+\left(\widetilde{K}^{2}\right)^{T} K+U,
$$

with the following initial condition:

$$
1=\sum_{m=0}^{M}(-1)^{m} X_{m}
$$

in which

$$
K=R+\mathscr{D}^{T} X
$$

and $\mathscr{D}$ is the operational matrix of delay given by (18). Moreover, $\widetilde{A}$ and $\widetilde{K}$ can be determined in a way similar to the construction method of the matrix $\widetilde{U}$ presented by (17). By solving (77)-(78), it follows that

$$
\begin{aligned}
X= & {\left[1,0,0,1,0,0, \frac{97}{75}, \frac{3}{10}, \frac{1}{150}, \frac{29}{15}, \frac{17}{50}, \frac{1}{150}, \frac{184}{75}, \frac{9}{50},\right.} \\
& \left.\frac{1}{150}\right]^{T} .
\end{aligned}
$$

Using (73), we would obtain the same value as the exact value of $x(t)$.

\section{Conclusion}

An efficient procedure has been successfully developed for solving delay differential equations with a piecewise constant delay function. The method is based upon a hybrid of blockpulse functions and Legendre polynomials. The nice properties of the hybrid functions together with the associated operational matrices were used to convert the original problem into a system of algebraic equations. The proposed framework allows one to simultaneously make use of the best advantages of the two mentioned bases. After determining the appropriate value of $N$, small values for $M$ are required to obtain an admissible approximation. It is worth noting that the correct choice of $N$ has a fundamental effect on the solution accuracy. It should be emphasized that the analytical solutions of Examples 1 and 3 cannot be derived solely either by block-pulse functions or by Legendre polynomials. The simulation results demonstrate the reliability and effectiveness of the proposed approximation scheme.

\section{Competing Interests}

The authors declare that they have no competing interests.

\section{References}

[1] M. Malek-Zavarei and M. Jamshidi, Time Delay Systems: Analysis, Optimization and Applications, North-Holland Systems and Control Series, Elsevier Science, New York, NY, USA, 1987.

[2] H. Smith, An Introduction to Delay Differential Equations with Applications to the Life Sciences, Texts in Applied Mathematics, Springer, Berlin, Germany, 2010.

[3] W. L. Chen and Y. P. Shih, "Shift Walsh matrix and delaydifferential equations," IEEE Transactions on Automatic Control, vol. 23, no. 6, pp. 1023-1028, 1978.

[4] W. L. Chen and B. S. Jeng, "Analysis of piecewise constant delay systems via block-pulse functions," International Journal of Systems Science, vol. 12, no. 5, pp. 625-633, 1981.

[5] A. Bellen, "One-step collocation for delay differential equations," Journal of Computational and Applied Mathematics, vol. 10, no. 3, pp. 275-283, 1984.

[6] A. Bellen and M. Zennaro, "Numerical solution of delay differential equations by uniform corrections to an implicit 
Runge-Kutta method," Numerische Mathematik, vol. 47, no. 2, pp. 301-316, 1985.

[7] A. Bellen and S. Maset, "Numerical solution of constant coefficient linear delay differential equations as abstract Cauchy problems," Numerische Mathematik, vol. 84, no. 3, pp. 351-374, 2000.

[8] Y. P. Shih, C. Hwang, and W. K. Chia, "Parameter estimation of delay systems via block pulse functions," Journal of Dynamic Systems, Measurement and Control, vol. 102, no. 3, pp. 159-162, 1980.

[9] F.-C. Kung and H. Lee, "Solution and parameter estimation in linear time-invariant delayed systems using laguerre polynomial expansion," Journal of Dynamic Systems, Measurement and Control, vol. 105, no. 4, pp. 297-301, 1983.

[10] L. Lee and F.-C. Kung, "Shifted Legendre series solution and parameter estimation of linear delayed systems," International Journal of Systems Science, vol. 16, no. 10, pp. 1249-1256, 1985.

[11] I. R. Horng and J. H. Chou, "Analysis, parameter estimation and optimal control of time-delay systems via Chebyshev series," International Journal of Control, vol. 41, no. 5, pp. 1221-1234, 1985.

[12] C. T. H. Baker, C. A. H. Paul, and D. R. Willé, "Issues in the numerical solution of evolutionary delay differential equations," Advances in Computational Mathematics, vol. 3, no. 3, pp. 171196, 1995.

[13] K. B. Datta and B. M. Mohan, Orthogonal Functions in Systems and Control, vol. 9 of Advanced Series in Electrical and Computer Engineering, World Scientific, Singapore, 1995.

[14] M. Razzaghi and H. R. Marzban, "A hybrid domain analysis for systems with delays in state and control," Mathematical Problems in Engineering, vol. 7, no. 4, pp. 337-353, 2001.

[15] H. R. Marzban and M. Razzaghi, "Solution of time-varying delay systems by hybrid functions," Mathematics and Computers in Simulation, vol. 64, no. 6, pp. 597-607, 2004.

[16] H. R. Marzban and M. Razzaghi, "Optimal control of linear delay systems via hybrid of block-pulse and Legendre polynomials," Journal of the Franklin Institute, vol. 341, no. 3, pp. 279293, 2004.

[17] H. R. Marzban and M. Razzaghi, "Analysis of time-delay systems via hybrid of block-pulse functions and Taylor series," Journal of Vibration and Control, vol. 11, no. 12, pp. 1455-1468, 2005.

[18] H. R. Marzban and M. Razzaghi, "Solution of multi-delay systems using hybrid of block-pulse functions and Taylor series," Journal of Sound and Vibration, vol. 292, no. 3-5, pp. 954-963, 2006.

[19] H. R. Marzban and M. Shahsiah, "Solution of piecewise constant delay systems using hybrid of block-pulse and Chebyshev polynomials," Optimal Control Applications \& Methods, vol. 32, no. 6, pp. 647-659, 2011.

[20] C. Canuto, M. Y. Hussaini, A. Quarteroni, and T. A. Zang, Spectral Methods: Fundamentals in Single Domains, Scientific Computation, Springer, Berlin, Germany, 2006.

[21] H. R. Marzban and S. M. Hoseini, "Numerical treatment of nonlinear optimal control problems involving piecewise constant delay," IMA Journal of Mathematical Control and Information, 2015.

[22] P. Lancaster, Theory of Matrices, Academic Press, New York, NY, USA, 1969.

[23] E. L. Ortiz, "The tau method," SIAM Journal on Numerical Analysis, vol. 6, no. 3, pp. 480-492, 1969. 


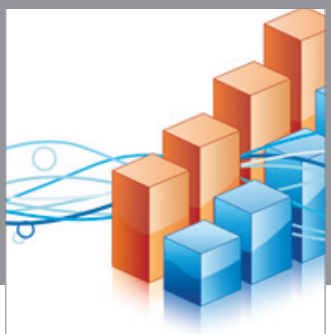

Advances in

Operations Research

vatem alat4

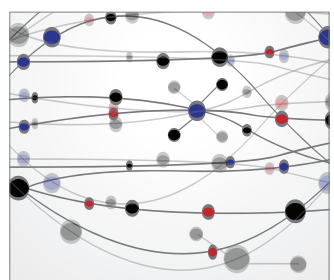

\section{The Scientific} World Journal
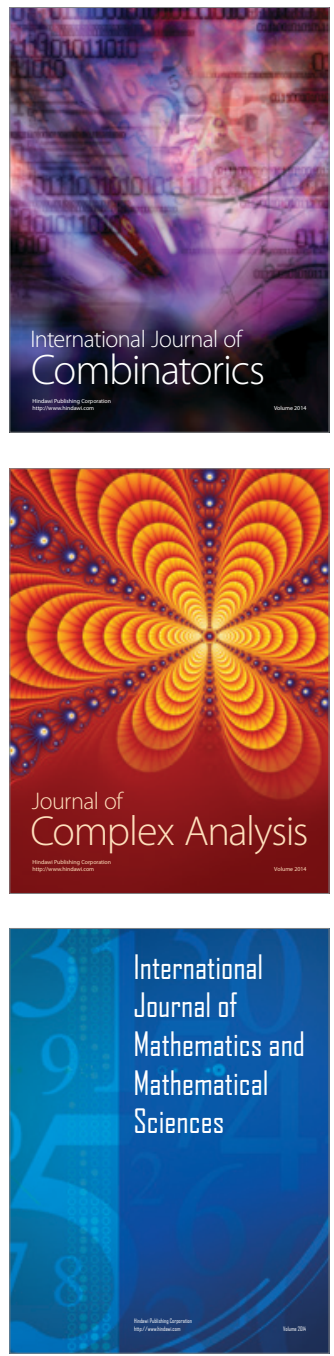
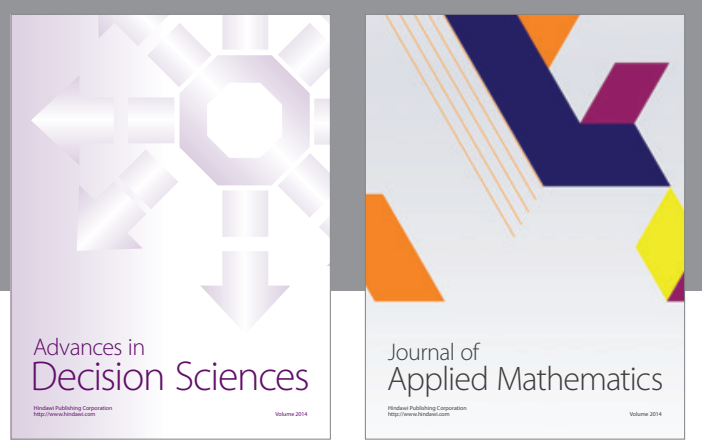

Algebra

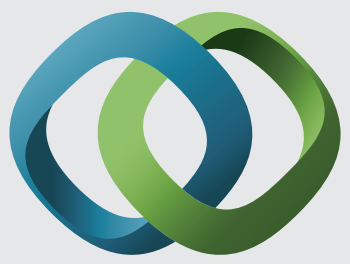

\section{Hindawi}

Submit your manuscripts at

http://www.hindawi.com
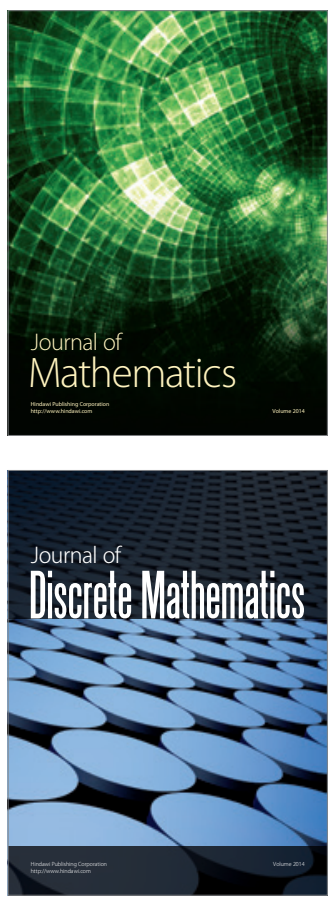

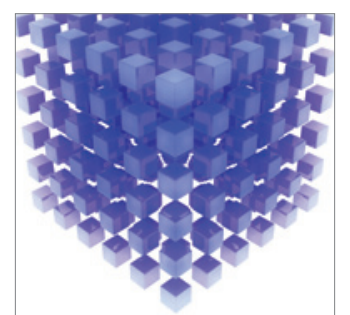

Mathematical Problems in Engineering
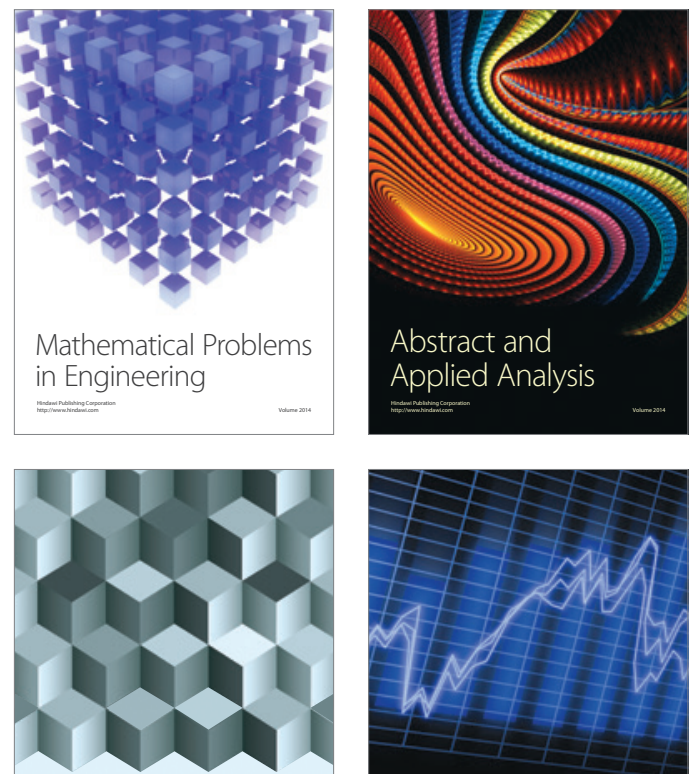

Journal of

Function Spaces

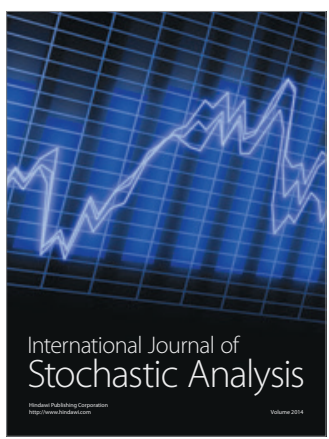

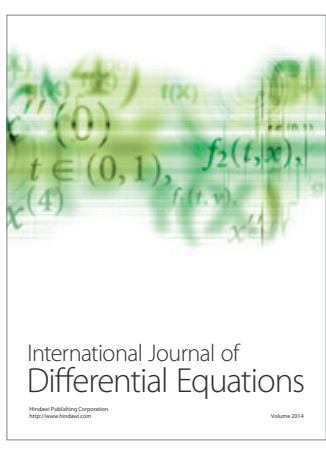
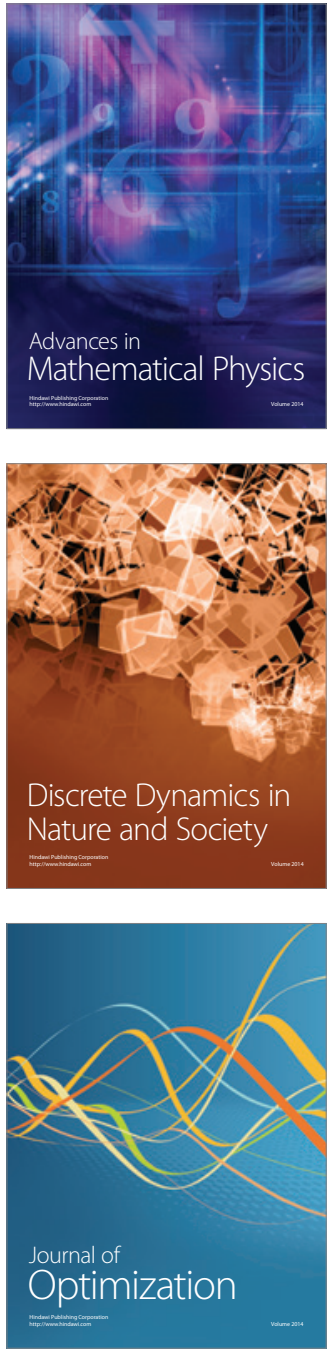